\title{
Impact of Interventions by a Community Pharmacist on Care Burden for People with Dementia: Development and Randomised Feasibility Trial of an Intervention Protocol
}

Yoko Nanaumi

Advance Pharma Research Office https://orcid.org/0000-0002-4680-9281

Atsushi Yoshitani

Nara Pharmaceutical Association

Mitsuko Onda ( $\nabla$ mitsuko.onda@ompu.ac.jp )

Osaka Medical and Pharmaceutical University

\section{Research}

Keywords: Dementia, caregiver burden, community pharmacists

Posted Date: September 23rd, 2021

DOI: https://doi.org/10.21203/rs.3.rs-917446/v1

License: @ (i) This work is licensed under a Creative Commons Attribution 4.0 International License. Read Full License 


\begin{abstract}
Background: Traditionally, the role of pharmacists has been to manage and monitor pharmacotherapy for patients with dementia. However, additional intervention by community pharmacists to collect and share patient information with other professionals may help reduce the care burden among caregivers. This study examined the impact of interventions by a community pharmacist, based on active information gathering from other professionals for people with dementia, on the caregivers' burden of care.
\end{abstract}

Methods: This was a randomised, open-label, parallel-group feasibility study, involving eight Nara City pharmaceutical association member pharmacies that provided consent to participate. These pharmacies were assigned to an intervention group or control group at a 1:1 ratio. The subjects were patients with dementia and their primary caregivers that visited the participating pharmacies and provided consent to participate. Pharmacists in the intervention group actively collected information from the patients' family physicians and care managers and intervened to address medication-related problems, while those in the control group only performed their normal duties. The primary endpoint was a change in the caregiver's score on the Japanese version of Zarit Caregiver Burden Interview (J-ZBI) from the baseline to after 5 months of follow-up. The changes in mean J-ZBI scores from the beginning to the end of the study period of the two groups were compared using Welch's t-test, and effect sizes were calculated. The level of significance was set at $5 \%$.

Results: Totally, nine patients and nine caregivers in the intervention group, and nine patients and eight caregivers in the control group completed the study. The changes in J-ZBI scores could be calculated for seven cases in the intervention group and five cases in the control group. The J-ZBI scores were found to decrease by 1.0 in the intervention group and increase by 3.0 in the control group $(p=0.075$, effect size $=1.414)$.

Conclusions: Although the differences in J-ZBI scores were not statistically significant between the two groups, owing to the small sample sizes, the effect sizes suggest that community pharmacist interventions for people with dementia may reduce the care burden for caregivers.

Trial registration: Registration: UMIN000039949 (registration date: 4.1.2020, retrospectively registered)

\title{
Key Message Regarding Feasibility \\ What uncertainties existed regarding the feasibility?
}

The Japanese National Strategy, which is a comprehensive strategy to accelerate dementia measures (New Orange Plan) in Japan, mentions that pharmacists represent the health professionals most accessible to the community. Therefore, the roles assigned to them include support for caregivers to reduce the burden of care. However, whether community pharmacist interventions contribute to a reduction in the caregiving burden is not fully understood.

\section{What are the key feasibility findings?}

Although the current study did not show statistically significant differences owing to the small sample size, the effect size suggests that active interventions by the community pharmacist may reduce the burden of care for the caregivers of people with dementia

\section{What are the implications of the feasibility findings for the design of the main study?}

The small sample size and short follow-up period represent limitations of this study. Additionally, interviews conducted with pharmacists after completion of the study revealed that while the presented protocol was believed to be feasible, the explanatory document provided to the pharmacists requires improvement. It was also noted that the intervention process requires simplification for implementation of a large-size study.

\section{Introduction}

It is estimated that more than 50 million people are currently living with dementia worldwide, a number predicted to increase to 152 million by 2050 [1]. In Japan alone, the number of dementia patients (prevalence rate) among the elderly over the age of 65 years is expected to reach 7 million (20\%) by 2025 [2], and the burden of care is becoming a social problem [3-6]. The lack of social services [7], low level of education of caregivers (intelligibility) [6], low selfefficacy of patients [8], behavioural and psychological symptoms of dementia (BPSD) [9], and psychosocial factors, including family functioning and relationships, contribute to the increased burden of care [10]. In general, caregivers of patients with dementia are prone to compromised health and low quality of life due to the physical, psychological, and social burdens associated with caregiving, resulting from the long process and unpredictable outcomes [11-13]. The increased burden of caregiving also has a profound impact on the well-being of society as a whole. Meanwhile, several interventional studies of field-level measures aimed at reducing the burden of caregiving have reported effectiveness [11], including improving relations between family and caregiving staff [12] and enhancing caregiver self-efficacy [13].

Several countries have national-level strategies, such as the Prime Minister's Challenge on Dementia in England [14], for supporting people with dementia through multi-profession collaborations [15]. Few, like the German [15] and Canadian national dementia strategies [16], specify the response to the burden of caregiving. However, the function and role of the pharmacy and pharmacist in reducing the care burden have not been clearly positioned in the inter-profession collaboration framework of either of these strategies.

The Japanese National Strategy is a comprehensive strategy formulated by the Ministry of Health, Labour and Welfare in July 2017 to accelerate dementia measures (New Orange Plan) in the country [17]. This plan has unique characteristics compared to the national strategies of other countries. For instance, the 
need for 'enhanced responsiveness to dementia' by pharmacies and pharmacists is specified in the context of a regional comprehensive care system. Specifically, since pharmacists represent the health professionals most accessible to the community [18], the roles assigned to them include support for caregivers to reduce the burden of care, early detection of dementia, and management of pharmacotherapy [17].

Traditionally, the primary roles of pharmacies and pharmacists in the care of people with dementia have been to improve the management of pharmacotherapy [19-22] and adherence to medication regimens [23, 24]. It has been suggested that community pharmacists can optimize pharmacotherapy for BPSD by sharing patient information with their general practitioners [25]. A prospective cohort study previously conducted by the authors suggests that enhancing the collection of patient information by community pharmacists may help to reduce the burden of caregiving [26]. In addition, assessing the effectiveness of community pharmacists by monitoring caregiver burden has been initiated [27]. However, whether community pharmacist interventions contribute to a reduction in the burden of caregiving is not fully understood [28]. Therefore, the objective of the current study was to evaluate whether active interventions by community pharmacists for people with dementia contribute to a reduction in the burden of caregiving.

\section{Methods Trial design}

The study was designed as a randomised, open-label, parallel-group, controlled feasibility study, and was performed in accordance with the CONSORT statement (pilot of feasibility randomised trial) [29]. In future studies, we plan to verify the effect of the intervention by conducting RCTs. Since there is no effect size that can be used as a reference in estimating the sample size, we would like to obtain a reference value in this pilot study.

\section{Participants}

\section{1) Pharmacies}

Member pharmacies of the Nara City pharmaceutical association who attended the briefing session for this study and agreed to participate were included as working pharmacies.

\section{2) Patients}

Outpatients with dementia who were using one of the research cooperation pharmacies and receiving anti-dementia medications were included in the study. Written informed consent was obtained from the patients or their legal representatives. Individuals who did not provide consent to participate, and those receiving home care services by pharmacists were excluded from the study. Home service recipients were excluded as their information may have been shared previously with the family physician and other related professionals, and interventions may have been advised accordingly.

\section{3) Caregivers}

The primary caregiver of a person with dementia provided consent to participate in the study. As a part of their job, community pharmacists are required to identify the primary caregiver while providing the medication; this information is recorded in the patient's profile. Accordingly, we obtained written consent from the primary caregiver to participate in the study when they visited the pharmacy.

\section{Randomisation}

Pharmacies were randomly assigned to a group by the study director according to a random computer-generated number table. Pharmacies were randomly assigned to the intervention or control group, in a 1:1 ratio, without employing block randomisation. A biostatistician at the Osaka University of Pharmaceutical Sciences employed computer-generated random numbers for obtaining the random allocation sequence. Pharmacists in each collaborative pharmacy recruited and assigned participants.

\section{Recruitment}

The participating pharmacists verbally introduced the program to those visiting the pharmacies. They approached those who were interested in the trial, explained its contents, and obtained written informed consent for their participation in the current trial. Participants were recruited from November 1-30, 2018; the follow-up period for each patient in the trial was at 5 months.

\section{Blinding}

The intervention was assumed to be behavioural; thus, the pharmacists were not blinded. However, the participants and analysts were blinded to group allocation.

\section{Intervention group}

Intervention group collected patient information during a patient/caregiver visit, using the 'Basic Information Sheet' shown in Appendix 1. The 'Basic Information Sheet' includes information collected during the first visit (patient's gender, age, primary caregiver, residential environment, prescriptions from medical institutions for the treatment of dementia, and prescriptions from other medical institutions), as well as information collected during each subsequent visit (details of any prescription changes made since the previous visit, and details of any actions taken by the family physician or care manager; Appendix 1 ). 
The main caregivers were also asked to complete the Japanese version of the Zarit Caregiver Burden Interview (J-ZBI) published by Sankyobo, (use registration number: $1494 \mathrm{~J}-\mathrm{ZBI}$ ). The J-ZBI is a self-administered questionnaire aimed at measuring a caregiver's perception of the burden of caregiving. The questionnaire is composed of 22 items, scored on a 5-point scale ranging from 0-4; the smaller the total score, the lower the burden of caregiving experienced by the individual [30]. In addition, the main caregiver was interviewed by the pharmacist based on the 'Understanding of dementia survey sheet' shown in Appendix 2. Scores were recorded as binary values (1: yes, 0: no). The survey sheet was a comprehension test used at Kishiwada-city in 2017 as the Dementia Supporter Training Program prepared by the Ministry of Health, Labour and Welfare. It consisted of five statements to be marked as yes or no: 1 . 'The progression of dementia can be slowed by treatment and care', 2. 'The progression of symptoms of dementia can be slowed down by medication', 3 . 'Dementia has "peripheral symptoms" in which behavioural and mental disorders occur', 4. 'There are several types of dementia, including Alzheimer's type and multi-infarct type', and 5. 'Measures against lifestyle related diseases are effective in preventing dementia'. The higher the total score, the better the caregiver's understanding of the condition (Appendix 2).

Next, pharmacists used the 'Grasp problems sheet' shown in Appendix 3 to interview the primary caregivers and assess any medication-related problems. Based on previous studies [31, 32], this sheet consisted of questions addressing the caregiver's awareness about the medication (6 items), collaboration with healthcare providers regarding medication ( 3 items), motivation to access and utilize information regarding medication ( 5 items), and agreement with patient taking medications and their fit with the patient's lifestyle ( 3 items). The aim was to identify and assess issues by setting assessment criteria for each question (Appendix 3).

The pharmacists sent forms for the request of information to the family physician (Appendix 4) and care manager (Appendix 5) to actively collect patient information. 'The form for request of information' delivered to the family physician asked for the name of the primary disease, information on clinical test data used for diagnosis, the level of cooperation with other occupations, guidance and treatment plan given to the family members and caregivers, and any requests for the pharmacy. A care manager is a qualified healthcare provider who develops a long-term care services plan and communicates and coordinates with care service providers upon request from the caregivers and families, as part of the Japanese long-term care insurance system implemented in 2000 . The request form to care managers asked for the main disease leading to the need for long-term care insurance service, degree of care required, results of care assessment and policy of care plan, level of cooperation with the family physician, problems with care and future policy, and any requests for the pharmacy (Appendix 4 and 5).

The pharmacist shared information with other professions, proposed prescription changes, changed dispensing methods, provided patient compliance instructions, requested consultations, and provided information on the issues identified in the 'Grasp problems sheet', based on information obtained in 'The forms for request of information'. The follow-up period was 5 months, and during the final visit, the patients/caregivers were interviewed again based on the Basic Information Sheet. The caregivers were asked to complete the J-ZBI and the 'Understanding of dementia survey sheet' once again.

\section{Control group}

Pharmacists in pharmacies assigned to the control group also collected information using the 'Basic Information Sheet', 'Understanding of dementia survey sheet', J-ZBI, and 'Grasp problems sheet.' However, they did not participate in aggressive information gathering using the 'Forms for request of information.'

\section{Outcomes}

1) The primary outcome was the change in the burden of care, and the primary endpoint was the change in the primary caregiver's J-ZBI score. 2) Secondary outcomes included changes in caregiver comprehension of dementia, prescription changes, and action taken by the attending physician or care manager. The endpoint for changes in the caregiver's understanding of the condition was the change in the primary caregiver's score in the 'Understanding of dementia survey sheet' (Appendix 2). The endpoint for prescription changes was the percentage of patients for whom the duration of a particular treatment was changed, or a medication was changed, added, or stopped. The endpoint for assessing the presence or absence of action taken by the family physician or care manager (excluding responding to the requests for information) was the percentage of patients who received an action from their family physician or care manager.

\section{Statistical analysis}

The mean differences between J-ZBI scores and dementia comprehension scores from the baseline to after 5 months of follow-up of the two groups were compared using Welch's t-test, followed by calculation of effect size (Cohen's d) [33]. In addition, the proportion of patients for whom prescription or care plans were changed was compared between the groups using the Chi-square test. Analysis was done at a significance level of $5 \%$. SPSS statistics software (IBM, Armonk, New York, USA) was used for analysis.

\section{Ethical considerations}

The study design was approved by the Research Ethics Review Committee of Osaka University of Pharmaceutical Sciences (approval number: 0048). Protocol registration ID is UMIN000039949. Figure 1 presents the study protocol for the intervention and control groups.

\section{Risk evaluation}

The interventions in this study were within the regular scope of pharmacy practice, and therefore, we assessed that no additional and fatal risks would arise from participation in the study.

\section{Results}




\section{Participants}

Of the 139 Nara City pharmaceutical association member pharmacies, 12 attended the study briefings and 8 provided consent to participate in the study. These pharmacies were allocated at a 1:1 ratio to the intervention and control groups. The purpose of the study was explained to the patients with dementia and caregivers visiting the participating pharmacies. Those who gave consent to participate were included in the study. Ten patients and caregivers were randomly assigned to the intervention group, and nine patients and eight caregivers were assigned to the control group (Fig. 2). Nine patients in each group completed the study during the survey period. In each group, two patients were male and seven were female. The primary caregivers included four sons, three daughters, one grandchild, and one spouse in the control group; and four sons, three daughters, one grandchild, and one patient in the intervention group (Table 1).

Table 1

Baseline demographic and clinical characteristics for each group

\begin{tabular}{|c|c|c|c|}
\hline Item & & IG $(n=9)$ & CG $(n=9)$ \\
\hline \multirow[t]{2}{*}{$\operatorname{Sex}(n)$} & Male & 2 & 2 \\
\hline & Female & 7 & 7 \\
\hline Age (years) & Mean (SD) & $86.1(5.4)$ & $84.0(6.6)$ \\
\hline \multirow[t]{5}{*}{ Primary caregivers $(\mathrm{n})$} & Patient & 0 & 1 \\
\hline & Spouse & 1 & 0 \\
\hline & Son & 4 & 4 \\
\hline & Daughter & 3 & 3 \\
\hline & Grandchild & 1 & 1 \\
\hline \multirow[t]{3}{*}{ Housing circumstances (n) } & Single residence & 3 & 4 \\
\hline & Living with Spouse & 1 & 1 \\
\hline & Living with a person other than the spouse & 5 & 4 \\
\hline Number of prescribed medicines (items) & Mean (SD) & $6.9(4.0)$ & $6.9(2.9)$ \\
\hline
\end{tabular}

\section{Outcomes}

$\mathrm{J}$-ZBI scores were obtained for the seven caregivers in the intervention group and five in the control group. The mean J-ZBI score in the intervention group decreased by 1.0 , and that in the control group increased by $3.0(p=0.075$, effect size $=1.414$; Table 2$)$. In the intervention group, J-ZBI scores improved in three subjects, remained unchanged in three, and worsened in one. Meanwhile, in the control group the J-ZBI score remained unchanged in two subjects and worsened in three. In the intervention group, the caregiver whose J-ZBI score worsened had a patient who was transitioning to tube feeding therapy during the study period. Of the 22 items evaluated in the $\mathrm{J}-\mathrm{ZBI}, 7$ scores decreased, and 8 scores increased, resulting in an overall worsening of 1 point.

Table 2

Comparison of outcomes in the two groups

\begin{tabular}{|c|c|c|c|c|c|c|c|c|c|}
\hline & \multicolumn{2}{|l|}{ Baseline } & \multicolumn{2}{|c|}{ After 5 months } & \multicolumn{2}{|c|}{ Change } & \multirow{3}{*}{$\begin{array}{l}P \\
\text { value }\end{array}$} & \multirow[t]{3}{*}{$95 \% \mathrm{Cl}$} & \multirow{3}{*}{$\begin{array}{l}\text { Cohen's } \\
\text { d }\end{array}$} \\
\hline & IG & CG & IG & CG & IG & CG & & & \\
\hline & Mean (SD) & Mean (SD) & Mean (SD) & Mean (SD) & $\begin{array}{l}\text { Mean } \\
(\mathrm{SD})\end{array}$ & $\begin{array}{l}\text { Mean } \\
\text { (SD) }\end{array}$ & & & \\
\hline J-ZBI score & $\begin{array}{l}24.57 \\
(14.85)\end{array}$ & $\begin{array}{l}15.60 \\
(13.83)\end{array}$ & $\begin{array}{l}23.57 \\
(15.11)\end{array}$ & $\begin{array}{l}18.60 \\
(13.22)\end{array}$ & $\begin{array}{l}-1.00 \\
(2.00)\end{array}$ & $\begin{array}{l}3.00 \\
(3.74)\end{array}$ & 0.075 & $\begin{array}{l}-0.563- \\
8.563\end{array}$ & 1.414 \\
\hline $\begin{array}{l}\text { Caregiver's understanding of } \\
\text { dementia }^{2}\end{array}$ & $4.11(1.05)$ & $3.13(1.81)$ & $4.44(1.01)$ & $2.62(2.13)$ & $\begin{array}{l}0.33 \\
(0.71)\end{array}$ & $\begin{array}{l}-0.51 \\
(1.07)\end{array}$ & 0.074 & $\begin{array}{l}-1.760- \\
0.093\end{array}$ & 0.932 \\
\hline
\end{tabular}

Note. ${ }^{1}$ Mean changes in scores (after 5 months - baseline) were calculated for the intervention group $(n=7)$ and the control group $(n=5)$, excluding those items that were not completed; ${ }^{2}$ Mean changes in scores (after 5 months - baseline) were calculated for the intervention group ( $n=9$ ) and the control group $(n=8)$, excluding those items that were not completed; There were no significant differences in J-ZBI and comprehension scores between the two groups at baseline $(P=0.414$ and 0.312 , respectively); IG: Intervention Group; CG: Control Group; SD: Standard Deviation; Cl: Confidence Interval; J-ZBI: the Japanese version of Zarit Caregiver Burden Interview.

Caregivers' dementia comprehension scores were obtained for nine participants in the intervention group and eight participants in the control group. The mean comprehension score increased by 0.33 in the intervention group and decreased by 0.51 in the control group $(p=0.074$, effect size $=0.932$; Table 2$)$. In the intervention group, the scores improved in two subjects, remained unchanged in seven subjects, and did not worsen in any subjects. In the control group, the scores did not improve in any subject, remained unchanged in six subjects, and worsened in two subjects. 
Prescription changes occurred for eight patients in the intervention group, versus four in the control group $(p=0.131$; Table 3$)$. Additionally, two patients from the intervention group received action from their family physician or care manager, while none from the control group received any action $(p=0.471$; Table 3 ).

Table 3

Prescription changes and actions taken

\begin{tabular}{|c|c|c|c|c|}
\hline & & IG & CG & $P$ value \\
\hline \multirow[t]{2}{*}{ Change in prescription } & Yes & 8 & 4 & 0.131 \\
\hline & No & 1 & 5 & \\
\hline \multirow[t]{2}{*}{ Actions taken by family physician and/or care manager } & Yes & 2 & 0 & 0.471 \\
\hline & No & 7 & 9 & \\
\hline
\end{tabular}

There were no adverse events associated with the interventions in this study.

\section{Discussion}

Although this study did not show statistically significant differences owing to the small sample size, the effect size suggests that active interventions by the community pharmacist may reduce the burden of care for caregivers of people with dementia. To discuss factors contributing to the reduction of care burden, we assessed the relevant information from three caregivers (Case 1-3) from the intervention group whose J-ZBI scores improved during the study (Table 4). 
Table 4

Summary of three cases in which the caregiving burden reduced

\begin{tabular}{|c|c|c|c|}
\hline & Case 1 (ID 429) & Case 2 (ID 4540) & Case 3 (ID 73570) \\
\hline $\begin{array}{l}\text { Primary } \\
\text { caregiver }\end{array}$ & Son & Grandchild & Spouse \\
\hline $\begin{array}{l}\text { Change in J-ZBI } \\
\text { scores }\end{array}$ & 51 to 50 & 15 to 10 & 31 to 29 \\
\hline \multirow[t]{5}{*}{$\begin{array}{l}\text { Items that } \\
\text { improved the J- } \\
\text { ZBI score* }\end{array}$} & $\begin{array}{l}\square \text { Afraid of what the future holds for } \\
\text { your relative }\end{array}$ & $\begin{array}{l}\text { QFeel that because of the time you spend with your } \\
\text { relative that you don't have enough time for yourself }\end{array}$ & $\begin{array}{l}\text { WFeel that you will be unable to } \\
\text { take care of your relative much } \\
\text { longer }\end{array}$ \\
\hline & & $\begin{array}{l}\text { पFeel stressed between caring for your relative and trying } \\
\text { to meet other responsibilities for your family or work }\end{array}$ & $\begin{array}{l}\text { WFeel you have lost control of } \\
\text { your life since your relative's } \\
\text { illness }\end{array}$ \\
\hline & & $\square$ Afraid of what the future holds for your relative & \\
\hline & & QFeel strained when you are around your relative & \\
\hline & & $\begin{array}{l}\text { पFeel you have lost control of your life since your relative's } \\
\text { illness }\end{array}$ & \\
\hline $\begin{array}{l}\text { Changes in } \\
\text { comprehension } \\
\text { scores }\end{array}$ & 5 to 5 & 3 to 5 & 4 to 5 \\
\hline \multirow{2}{*}{$\begin{array}{l}\text { Items that } \\
\text { improved in } \\
\text { comprehension } \\
\text { score }^{\star}\end{array}$} & & पUnderstand that there are several types of dementia & $\begin{array}{l}\text { qunderstand that there are } \\
\text { several types of dementia }\end{array}$ \\
\hline & & qUnderstand that dementia has peripheral symptoms & \\
\hline \multirow{7}{*}{$\begin{array}{l}\text { Problems } \\
\text { identified } \\
\text { by the } \\
\text { pharmacist } \\
\text { from the Grasp } \\
\text { problem sheet* }\end{array}$} & $\begin{array}{l}\text { पTake medication at one's own } \\
\text { discretion or not at all }\end{array}$ & $\square$ Not coping with continued medications & $\begin{array}{l}\text { Dissatisfied with the current } \\
\text { dosing regimen for the rest of } \\
\text { their life }\end{array}$ \\
\hline & $\begin{array}{l}\text { QDiscontinue the medication at your } \\
\text { own discretion when your condition } \\
\text { changes }\end{array}$ & & $\begin{array}{l}\text { aLack of care for any changes in } \\
\text { the patient's condition while } \\
\text { taking the medicines }\end{array}$ \\
\hline & $\begin{array}{l}\text { Not being able to ask questions } \\
\text { about medications to healthcare } \\
\text { providers without hesitation }\end{array}$ & & $\begin{array}{l}\text { Not finding and using the } \\
\text { information needed for the } \\
\text { patient's medication }\end{array}$ \\
\hline & $\begin{array}{l}\text { Not finding and using the } \\
\text { information needed for the patient's } \\
\text { medication }\end{array}$ & & $\begin{array}{l}\text { Not asking when you don't know } \\
\text { about the medication the } \\
\text { patient is using }\end{array}$ \\
\hline & $\begin{array}{l}\text { aNot coping with continued } \\
\text { medications }\end{array}$ & & \\
\hline & $\begin{array}{l}\text { पNot knowing about the medication } \\
\text { used by patient and why they are } \\
\text { needed. }\end{array}$ & & \\
\hline & $\begin{array}{l}\text { पNot asking when you don't know } \\
\text { about the medication the patient is } \\
\text { using }\end{array}$ & & \\
\hline $\begin{array}{l}\text { Reply to the } \\
\text { form for request } \\
\text { of information }\end{array}$ & $\begin{array}{l}\text { Reply from family physician and } \\
\text { care manager }\end{array}$ & Reply from family physician and care manager & $\begin{array}{l}\text { Reply from family physician } \\
\text { and care manager }\end{array}$ \\
\hline \multirow{2}{*}{$\begin{array}{l}\text { Actions by the } \\
\text { family physician } \\
\text { or care } \\
\text { manager }\end{array}$} & $\begin{array}{l}\text { The family physician instructed the } \\
\text { pharmacist to visit the patient } \\
\text { residence. }\end{array}$ & $\begin{array}{l}\text { पCare manager asked pharmacists to participate in care } \\
\text { conferences. }\end{array}$ & $\begin{array}{l}\text { QFamily physician contacted } \\
\text { separately }\end{array}$ \\
\hline & & $\begin{array}{l}\text { पCare manager directly contacted. There was an increase } \\
\text { in direct contact from care managers to pharmacists ( } 3 \\
\text { times during the study period). }\end{array}$ & \\
\hline \multirow{2}{*}{$\begin{array}{l}\text { Details of the } \\
\text { prescription } \\
\text { change* }^{*}\end{array}$} & Addition of antitussives & aDonepezil dose increased from $3 \mathrm{mg}$ to $5 \mathrm{mg}$. & $\begin{array}{l}\text { LLinaclotide and mirabegron } \\
\text { tablets were discontinued. }\end{array}$ \\
\hline & & & $\begin{array}{l}\text { पChanged from internal } \\
\text { vicosulfate solution to tablet }\end{array}$ \\
\hline
\end{tabular}


In Case 1, the pharmacist identified problems related to the 'awareness of medication', 'collaboration with healthcare providers regarding medication', and 'motivation to access and utilize information regarding medication', including discontinuing medication at own direction, not being able to ask questions about medications to healthcare providers without hesitation, and not knowing about the medication used by the patient and why it is required. This information was shared with the family physician and care manager via 'The form for request of information', and the pharmacist was subsequently instructed to visit the patient's residence. As a result, it became possible for the pharmacist to provide the necessary information. For instance, they were able to discuss the need and policy for the patient's specific pharmacotherapy as well as the features of the additional medication; in response, the pharmacist was provided the opportunity to respond to the caregiver's questions. These interactions may have served to reduce caregiver anxiety as it would be made clear that the necessary support would be available when required, while also providing the pharmacist with a better understanding of patient and caregiver living circumstances, allowing them to tailor the patient's care accordingly.

In Case 2, the pharmacist identified problems related to 'motivation to access and utilize information regarding medication', particularly related to continuation of medication. Subsequently, through information sharing via 'The form for request of information', the frequency of contact between the care manager and pharmacist increased, leading to pharmacist participation in the care conference of the patient in question. Care conferences are organized by care managers and include the gathering of healthcare providers and caregivers to identify specific issues and requests related to the patient's medical care, while assessing the support measures. During the care conference, the pharmacist involved in the patient's care is afforded the opportunity to respond directly to the caregiver's questions and concerns, thus, improving the caregiver's understanding of dementia. The conference also allows caregivers to experience the benefits of a multi-profession support system in the care of the patient, as well as the support system's capacity to potentially reduce stress and anxiety associated with care. Additionally, in this specific case, treatment with donepezil was effectively continued after a prescription change. This discussion is also supported by previous reports claiming that the addition of pharmacists to care teams for people with dementia leads to a reduction in the care burden for caregivers [34].

In Case 3, issues related to 'awareness of medication' and 'motivation to access and utilize information regarding medication' were identified, including dissatisfaction with the current dose for the rest of the patient's life, lack of regard for any changes in the patient's condition while taking the medicine, not finding and using the information needed for medication, and not asking questions when not knowing what the patient is using. Subsequently, information obtained through the 'The form for request of information' increased the frequency of contact between the family physician and the pharmacist and revealed that the patient was reluctant to use the patch medication, while the caregiver believed that there were no side effects to it. No changes were made to this prescription during the survey period; however, two other medications were discontinued, and the route for administering constipation medication was changed for ease of management. Enhanced collaboration between the physician and pharmacist may have improved the caregivers' comprehension of dementia and reduced the caregivers' sense of constraint due to the care provided. These findings were consistent with previous reports that have indicated that effective physician-pharmacist communication contributes to a better understanding of patient needs [35], while information sharing among families, physicians, care managers, and other professionals involved in care contributes to a reduction in the care burden [36].

In the three case studies discussed, we believe that the active information gathering from other professionals conducted by the pharmacists in the intervention group enabled the identification and individualization of problems contributing to the burden of care and improved the caregivers' comprehension of medication. Both factors contributed to the reduction in the caregiving burden. This conclusion is supported by the caregivers' improved comprehension of the dementia medication, allowing for better medication control and improved medication adherence [37], and by the reported increase in the caregivers' selfefficacy and reduction in the burden of caregiving [38-40]. Meanwhile, it has also been reported that psychological interventions for caregivers by healthcare professionals contribute to reduced care burden [41,42]. Therefore, in the future, it is important for pharmacists to actively conduct information gathering for people with dementia, cooperate with other professionals, share information, and conduct assessments. It will also be important for pharmacists to gain familiarity with programs to support self-care for caregivers, such as cognitive behavioural therapy, and to seek and practice communication and engagement with caregivers.

However, the small sample size and short follow-up period represent limitations of this study. Additionally, interviews conducted with pharmacists after study completion revealed that while the presented protocol was believed to be feasible, the explanatory document provided to the pharmacists requires improvement. Specifically, a more thorough explanation regarding the study design and commitments is required for the pharmacies to provide consent for participation. It was also noted that the intervention process requires simplification. Accordingly, the proposed changes include simplifying the methods by which data is collected, using a shorter version of the J-ZBI (J-ZBI 8) [43], and prolonging the follow-up period (e.g., matching the care manager to the 6-month interval between periodic reviews of the care plan).

\section{Generalizability (applicability) of pilot trial methods and findings to future definitive trial and other studies}

The feasibility of this trial was verified. However, large-scale studies are required for further standardization and simplification of the protocol to generalize these results. In addition, accurate outcome measures need to be developed to evaluate the effectiveness and wider application of these interventions. Thus, although this trial focused on dementia, we believe that the protocol can be restructured to be applied to other diseases.

\section{Conclusion}

Active intervention by community pharmacists for patients with dementia is important not only for the appropriateness of medication therapy, but also for its potential contribution to improving the quality of life of patients and caregivers by reducing the burden of care. This was a preliminary study, and due to the low sample size, we were not able to show statistically significant differences in J-ZBI scores between the two groups, although we were able to obtain sufficient effect sizes. In the future, we plan to conduct a study with a larger number of participants to confirm the results of the current study. 


\section{Abbreviations}

IG: Intervention group; CG: Control group; SD: standard deviation; J-ZBI: Japanese version of the Zarit Caregiver Burden Interview; FP: Family physician

\section{Declarations}

\section{Ethics approval and consent to participate}

The study design was approved by the Research Ethics Review Committee of Osaka University of Pharmaceutical Sciences (approval number: 0048), Protocol registration ID: UMIN000039949. Consent to participate was obtained from all study participants.

https://rctportal.niph.go.jp/s/detail/um?trial_id=UMIN000039949

\section{Consent for publication}

Not applicable.

\section{Availability of data and materials}

The datasets used and/or analysed during the current study are available from the corresponding author on reasonable request.

\section{Competing interests}

The authors declare that they have no competing interests.

\section{Funding}

This research received no specific grant from any funding agency in the public, commercial, or not-for-profit sectors.

\section{Authors' contributions}

YN conceived the study, collected the data, and wrote the paper. AY secured the research field, conducted the research, and managed the progress. MO conceived and designed the study, made important revisions to the paper, and approved the final draft. All authors read and approved the final manuscript.

\section{Acknowledgements}

We appreciate the contributions of the Nara City pharmaceutical association and the pharmacists who cooperated with them. Also, we would like to thank Editage (www.editage.com) for English language editing.

\section{References}

1. Alzheimer's Disease International. World Alzheimer Report 2019. https://www.alzint.org/u/WorldAlzheimerReport2019.pdf. Accessed 29 Apr 2019.

2. Cabinet Office Japan. Annual Report on the Ageing Society [Summary] FY 2019. https://www8.cao.go.jp/kourei/english/annualreport/2019/pdf/2019.pdf. Accessed 29 Apr 2019.

3. Cheng ST. Dementia caregiver burden: a research update and critical analysis. Curr Psychiatry Rep. 2017;19:64. doi:10.1007/s11920-017-0818-2.

4. Chiao CY, Wu HS, Hsiao CY. Caregiver burden for informal caregivers of patients with dementia: a systematic review. Int Nurs Rev. 2015;62:340-50. doi:10.1111/inr.12194.

5. World Health Organization. Towards a dementia plan: a WHO guide. 2018. https://apps.who.int/iris/bitstream/handle/10665/272642/9789241514132eng.pdf?

ua=1\#: :text=Towards\%20a\%20dementia\%20plan\%3A\%20a\%20WHO\%20guide\%20provides\%20technical\%20guidance,into\%20existing\%20dementia\%2D Accessed 29 Apr 2019.

6. Hughes TB, Black BS, Albert M, Gitlin L, Johnson D, Lyketsos C, et al. Correlates of objective and subjective measures of caregiver burden among dementia caregivers: influence of unmet patient and caregiver dementia-related care needs. Int Psychogeriatr. 2014;26:1875-83. doi:10.1017/S1041610214001240.

7. Naganuma Y, Kihara S, Fujita Y, Yamaoka K, Takahashi K. The relation between social cohesion and the care burden of family healthcare providers. BioSci Trends. 2018;12:520-2. doi:10.5582/bst.2018.01175.

8. Gilliam CM, Steffen AM. The relationship between caregiving self-efficacy and depressive symptoms in dementia family caregivers. Aging Ment Health. 2006;10:79-86. doi:10.1080/13607860500310658. 
9. Vaingankar JA, Chong SA, Abdin E, Picco L, Jeyagurunathan A, Zhang YJ, et al. Care participation and burden among informal caregivers of older adults with care needs and associations with dementia. Int Psychogeriatr. 2016;28:221-31. doi:10.1017/S104161021500160X.

10. Saeki A. The influence of family functions and family systems on primary caregiver burden caring for elderly people with dementia. The Japanese Red Cross Kyushu International College of Nursing 2006; 5:55-62. https://jrckicn.repo.nii.ac.jp/?

action=pages_view_main\&active_action=repository_view_main_item_detail\&item_id=95\&item_no=1\&page_id=13\&block_id=17. Accessed 14 Jun 2020 .

11. Só§rensen S, Pinquart M, Duberstein P. How effective are interventions with caregivers? An updated meta-analysis. Gerontologist. 2002;42:356-72. doi:10.1093/geront/42.3.356.

12. Nguyen M, Pachana NA, Beattie E, Fielding E, Ramis MA. Effectiveness of interventions to improve family-staff relationships in the care of people with dementia in residential aged care: a systematic review protocol. JBI Database System Rev Implement Rep. 2015;13:52-63. doi:10.11124/jbisrir-20152415.

13. Gallagher D, Ni Mhaolain A, Crosby L, Ryan D, Lacey L, Coen RF, et al. Self-efficacy for managing dementia may protect against burden and depression in Alzheimer's caregivers. Aging Ment Health. 2011;15:663-70. doi:10.1080/13607863.2011.562179.

14. Department of Health. Prime Ministers Challenge on Dementia.

2020. https://assets.publishing.service.gov.uk/government/uploads/system/uploads/attachment_data/file/507981/PM_Dementia-main_acc.pdf. Accessed 29 Apr 2021.

15. Alzheimer Europe. Policy: National dementia strategies. https://www.alzheimer-europe.org/Policy/National-Dementia-Strategies. Accessed 9 Jul 2020.

16. Government of Canada. A dementia strategy for Canada: together we aspire: in brief. 2019. https://www.canada.ca/en/publichealth/services/publications/diseases-conditions/dementia-strategy-brief.html. Accessed 26 Jul 2020.

17. Ministry of Health and Welfare. Japanese dementia strategy (new orange plan). 2017. https://www.mhlw.go.jp/file/06-Seisakujouhou-12300000Roukenkyoku/kaitei_orangeplan.pdf. Accessed 24 May 2021.

18. Cotelo NV, Rodríguez NFA, Pérez JAF, Iglesias JCA, Lago MR. Burden and associated pathologies in family caregivers of Alzheimer's disease patients in Spain. Pharm Pract. 2015;13:521. doi:10.18549/pharmpract.2015.02.521.

19. Goedken AM, Huang S, McDonough RP, Deninger MJ, Doucette WR. Medication-related problems identified through continuous medication monitoring. Pharmacy. 2018;6:86. doi:10.3390/pharmacy6030086.

20. While C, Duane F, Beanland C, Koch S. Medication management: the perspectives of people with dementia and family carers. Dementia. 2013;12:734-50. doi:10.1177/1471301212444056.

21. Marasco R, Heely JA, Gardner M. The pharmacist's role in managing patients with Alzheimer's disease. J Am Pharm Assoc. 2003;43:20-1. doi:10.1331/154434503322612339.

22. Nakamura K, Watanabe N, Ohkawa H, Ando M, Ogura Y, Funabiki S, et al. Effects on caregiver burden of a donepezil hydrochloride dosage increase to 10 $\mathrm{mg} /$ day in patients with Alzheimer's disease. Patient Prefer Adherence. 2014;8:1223-8. doi:10.2147/PPA.S69750.

23. Kröger E, Tatar $\mathrm{O}$, Vedel I, Giguère AMC, Voyer P, Guillaumie L, et al. Improving medication adherence among community-dwelling seniors with cognitive impairment: a systematic review of interventions. Int J Clin Pharm. 2017;39:641-56. doi:10.1007/s11096-017-0487-6.

24. Hudani ZK, Rojas-Fernandez CH. A scoping review on medication adherence in older patients with cognitive impairment or dementia. Res Social Adm Pharm. 2016;12:815-29. doi:10.1016/j.sapharm.2015.11.011.

25. Maidment ID, Aston L, Hilton A, Iqbal N, Child A, Shaw R. Role of community pharmacists in the use of antipsychotics for behavioural and psychological symptoms of dementia (BPSD): a qualitative study. BMJ Open. 2016;6:e010278. doi:10.1136/bmjopen-2015-010278.

26. Nanaumi Y, Onda M, Tsubota K, Tanaka R, Mukai Y, Matoba S, et al. Effectiveness of pharmacists' comprehensive assessment of medication profiles in dementia patients. Yakugaku Zasshi. 2015;135:1057-67. doi:10.1248/yakushi.15-00109.

27. Novais T, Mouchoux C. Pharmaceutical care for patients and their caregivers in Alzheimer's disease and related disorders: a review and new perspectives. Geriatr Psychol Neuropsychiatr Vieil. 2019;17:7-19. doi:10.1684/pnv.2018.0767.

28. Dann-Reed E, Poland F, Wright D. Systematic review to inform the development of a community pharmacy-based intervention for people affected by dementia. Int J Pharm Pract. 2020;28:233-45. doi:10.1111/ijpp.12586.

29. Eldridge SM, Chan CL, Campbell MJ, Bond CM, Hopewell S, Thabane L, et al. CONSORT 2010 statement: extension to randomised pilot and feasibility trials. BMJ. 2016;355:i5239. doi:10.1136/bmj.i5239.

30. Arai Y, Kudo K, Hosokawa T, Washio M, Miura H, Hisamichi S. Reliability and validity of the Japanese version of the Zarit Caregiver Burden interview. Psychiatry Clin Neurosci. 1997;51:281-7. doi:10.1111/j.1440-1819.1997.tb03199.x.

31. Ueno H, Yamazaki Y, Ishikawa H. Reliability and validity of medication adherence scale for patients with chronic disease in Japan. Jap J Health Educ Promot. 2014;22:13-29.

32. Kubo T, Kaji M, Tsuji S, Asakura M, Higuchi K, Mukai E, et al. Improvement of method to estimate guidance by pharmacists and trial to obtain standard pharmaceutical management and guidance services program. Yakugaku Zasshi. 2001;121:221-32. doi:10.1248/yakushi.121.221.

33. Sullivan GM, Feinn R. Using effect size-or why the $P$ value is not enough. J Grad Med Educ. 2012;4:279-82. doi:10.4300/JGME-D-12-00156.1.

34. Bernstein A, Merrilees J, Dulaney S, Harrison KL, Chiong W, Ong P, et al. Using care navigation to address caregiver burden in dementia: A qualitative case study analysis. Alzheimer's Dement. 2020;6:e12010. doi:10.1002/trc2.12010.

35. Weissenborn M, Haefeli WE, Peters-Klimm F, Seidling HM. Interprofessional communication between community pharmacists and general practitioners: a qualitative study. Int J Clin Pharm. 2017;39:495-506. doi:10.1007/s11096-017-0450-6. 
36. Sato S, Kazui H, Shimizu Y, Yoshida T, Yoshiyama K, Kanemoto H, et al. Usefulness of carer-held records to support informal caregivers of patients with dementia who live at home. Psychogeriatrics. 2018;18:166-74. doi:10.1111/psyg.12304.

37. Nanaumi Y, Onda M, Mukai Y, Tanaka R, Tubota K, Matoba S, et al. Factors related to medication adherence of cognitively impaired patients in community pharmacies. Pharmacol Pharm. 2012;3:368-80. doi:10.4236/pp.2012.33050.

38. Sakurai N. The moderating effects of positive appraisal on the burden of family caregivers of older people. Shinrigaku Kenkyu. 1999;70:203-10. doi:10.4992/jjpsy.70.203.

39. El-Saifi N, Moyle W, Jones C. Family caregivers' perspectives on medication adherence challenges in older people with dementia: a qualitative study. Aging Ment Health. 2019;23:1333-9. doi:10.1080/13607863.2018.1496226.

40. Adler G, Mueller B, Articus K. The transdermal formulation of rivastigmine improves caregiver burden and treatment adherence of patients with Alzheimer's disease under daily practice conditions. Int J Clin Pract. 2014;68:465-70. doi:10.1111/ijcp.12374.

41. Qiu D, Hu M, Yu Y, Tang B, Xio S. Acceptability of psychosocial interventions for dementia caregivers: a systematic review. BMC Psychiatry. $2019 ; 19: 23$. doi:10.1186/s12888-018-1976-4.

42. Teahan Á, Lafferty A, McAuliffe E, Phelan A, O'Sullivan L, O'Shea D, et al. Psychosocial interventions for family carers of people with dementia: a systematic review and meta-analysis. J Aging Health. 2020;32:1198-213. doi:10.1177/0898264319899793.

43. Kumamoto K, Arai Y. Validation of 'personal strain' and 'role strain': subscales of the short version of the Japanese version of the Zarit Burden Interview (JZBI_8). Psychiatry Clin Neurosci. 2004; 58:606-10. doi:10.1111/j.1440-1819.2004.01310.x

\section{Figures}

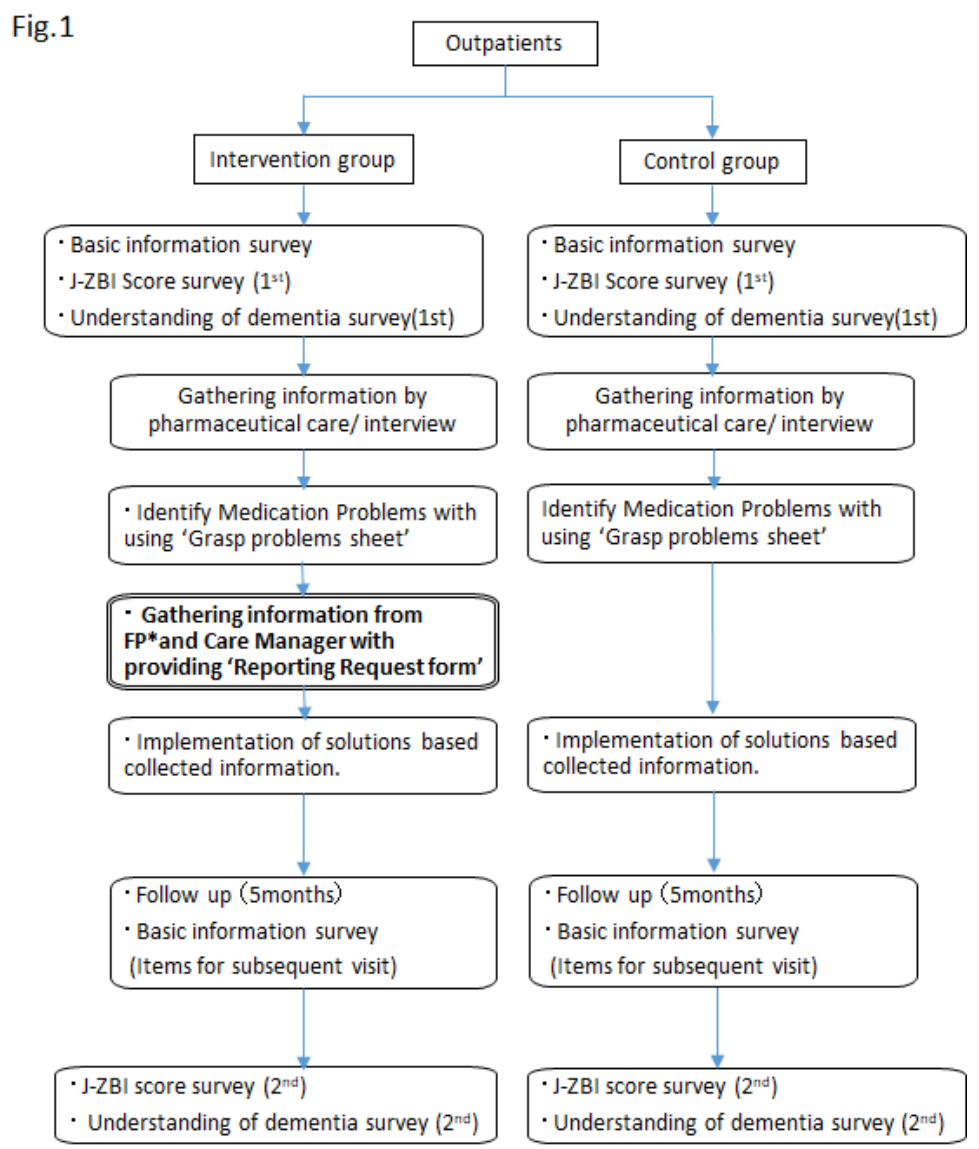

Study Protocol in two groups

\section{Figure 1}

Study protocol for the two groups 
Fig.2

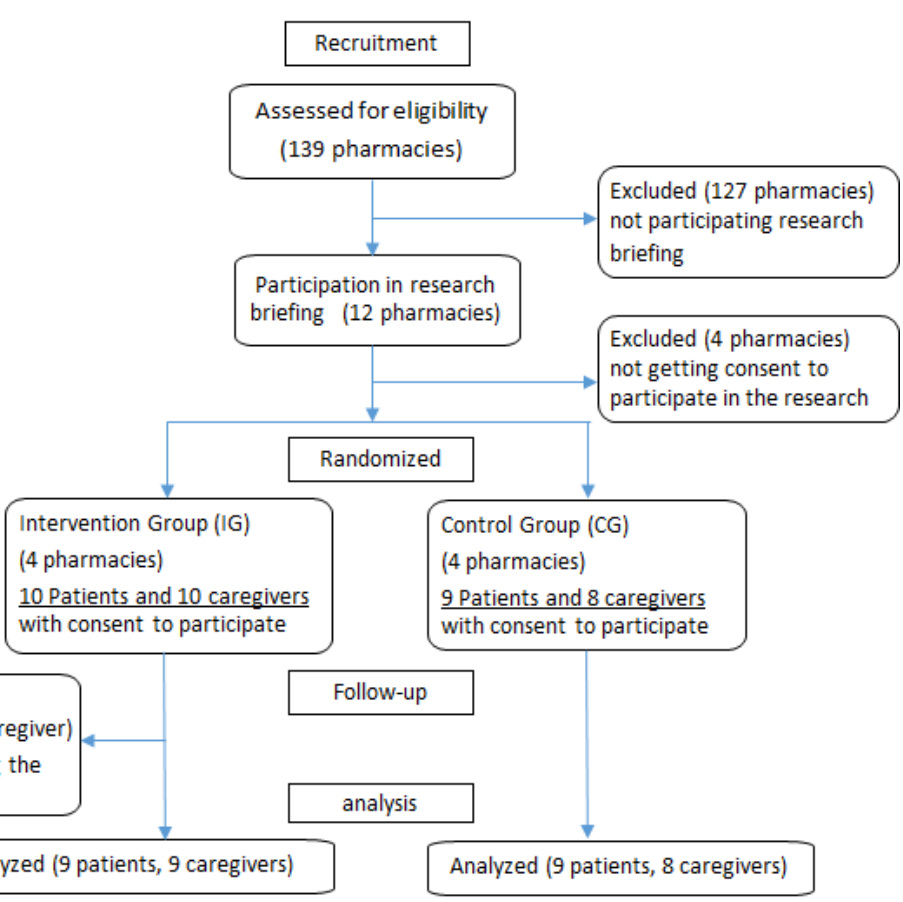

Consort flow of Participants

Figure 2

Consort flowchart of participant selection

\section{Supplementary Files}

This is a list of supplementary files associated with this preprint. Click to download.

- CONSORTforPilotandFeasibilityTrialsChecklist.doc

- supplementalyokonanaumiver.27.docx 